\title{
Large-scale structure-based prediction and identification of novel protease substrates using computational protein design
}

Manasi A. Pethe1,4, Aliza B. Rubenstein ${ }^{2,3}$ \& Sagar D. Khare*1,2,3,4 
Abstract: Characterizing the substrate specificity of protease enzymes is critical for illuminating the molecular basis of their diverse and complex roles in a wide array of biological processes. Rapid and accurate prediction of their extended substrate specificity would also aid in the design of custom proteases capable of selectively and controllably cleaving biotechnologically or therapeutically relevant targets. However, current in silico approaches for protease specificity prediction, rely on, and are therefore limited by, machine learning of sequence patterns in known experimental data. Here, we describe a general approach for predicting peptidase substrates de novo using protein structure modeling and biophysical evaluation of enzyme-substrate complexes. We construct atomic resolution models of thousands of candidate substrate-enzyme complexes for each of five model proteases belonging to the four major protease mechanistic classes - serine-, cysteine-, aspartyl- and metallo-proteases, and develop a discriminatory scoring function using enzyme design modules from Rosetta and Amber-MMPBSA. We rank putative substrates based on calculated interaction energy with a modeled near-attack conformation of the enzyme active site. We show that the energetic patterns obtained from these simulations can be used to robustly rank and classify known cleaved and uncleaved peptides and that these structural-energetic patterns have greater discriminatory power compared to purely sequence-based statistical inference. Combining sequence and energetic patterns using machine-learning algorithms further improves classification performance, and analysis of structural models provides physical insight into the structural basis for the observed specificities. We further tested the predictive capability of the model by designing and experimentally characterizing the cleavage of four novel substrate motifs for the Hepatitis C virus NS3/4 protease using an in vivo assay. The presented structure- 
based approach is generalizable to other protease enzymes with known or modeled structures, and complements existing experimental methods for specificity determination.

\author{
Abbreviations \\ TEV, Tobacco Etch Virus Protease, HCV, Hepatitis C Virus Protease, HIVPR, Human \\ Immunodeficiency Virus Protease 1, AUC, Area Under Curve, ROC, Receiver Operator Curve, \\ SVM, Support Vector Machine, YESS Yeast Endoplasmic Reticulum Sequestration and \\ Screening, TI, tetrahedral intermediate
}

\title{
Keywords
}

Proteases, substrate specificity, computational modeling, Rosetta software, specificity prediction, protease-peptide interactions

\section{Introduction}

Proteolytic cleavage is a ubiquitous post-translational modification that controls the transmission of biological information(1-3). Proteases encompass a structurally and mechanistically diverse class of enzymes that display a range of cleavage specificities reflecting their complex and diverse biological roles (3-6). For example, proteases involved in digestion and extracellular matrix degradation, e.g. trypsins and matrix metalloproteases, respectively, show relatively relaxed specificity profiles(7), whereas those involved in apoptotic and thrombolytic cascades, e.g. caspases(8) and thrombin(9), respectively, are more selective in their cleavage motifs. In many viruses, protease- 
mediated cleavage of the viral polyprotein at specific sites is crucial for viral maturation(10); as a result, these enzymes are highly selective in cleaving only a small set of polypeptide sequences, while not acting on other sequences in the polyprotein. Accordingly, these enzymes have been successful drug targets for developing anti-viral therapies $(11,12)$. Thus, proteases are exemplars of enzymatic multi-specificity, which have likely evolved to act upon and cleave a range of substrates - their specificity profile while simultaneously avoiding the cleavage of other substrates (13). Modeling of protease substrate specificity would illuminate the structural and physiochemical basis of these observed positive and negative selectivities, and aid protease biology by identifying novel substrates and biological roles of proteolysis.

Experimental methods to characterize protease specificity (14) range from low-throughput methods in which individual peptides or mixtures of peptides are assayed for cleavage(1517) to high-throughput methods that allow identification of substrates on a proteome-wide scale $(8,18-21)$. However, substrate sequence space is large and different proteome-wide datasets often have little overlap, suggesting that a large number of substrate sequences remain to be identified. Moreover, each experiment is limited to a single enzyme variant (typically the wild type). Computational approaches could, in principle, enable more rapid construction of specificity profiles, especially for naturally occurring or drug-resistant protease variants, and/or assist in library design for experimental specificity determination in a specific region of sequence space. Pattern recognition-based approaches have been used to predict substrate sequence preferences for various proteases based on machine learning from available experimental data (22-27). However, these sequence-only 
approaches are constrained by the quality of the input data, and cannot be generalized to other proteases, or to variants of the same protease enzyme.

Proteolysis is a multi-step reaction involving the binding of the substrate and subsequent nucleophilic attack on the carbonyl group carbon of the scissile peptide bond to yield a tetrahedral intermediate (TI; Fig. 1A-C) (2). Steps after TI formation are mechanismdependent: in cysteine, serine (and threonine) proteases, the intermediate disproportionates to yield one product and the reaction proceeds via the formation of an enzyme-bound intermediate that is deacylated to yield the second product (Fig. 1A). In aspartic (and glutamic), and metallo-proteases, which use a hydroxide nucleophile generated from a bound water molecule, the tetrahedral intermediate directly disproportionates into both products (Fig. 1B,C). In principle, different steps could determine substrate specificity depending on the substrate and the mechanism under consideration. However, for all proteases, regardless of the mechanistic class they belong to, the first step, i.e., enzyme nucleophilic attack is required for turnover (2). This observation led us to hypothesize that a model of the enzyme with the bound substrate and catalytic machinery modeled in a near-nucleophilic attack conformation would enable us to capture the energetics involved in substrate recognition and specificity.

Here, we develop a predictive biophysical model aimed at uncovering the underlying rules that govern protease-peptide molecular recognition and test its ability to classify known protease substrates from uncleaved ones. We construct a discriminative scoring function that includes descriptors of the energetics (including long-range electrostatic interactions) 


\section{Results}

\section{Rationale for the Curation of Benchmark Datasets:}

To develop and test a general structure- and energy-based prediction approach for protease specificity, we curated benchmark sequence sets for five protease enzymes. These exhibit diverse mechanisms of action, varied folds and biological functions - TEV Protease (cysteine proteases), HCV NS3 protease (serine proteases), Granzyme B (serine protease), HIV Protease-1 (aspartyl protease) and Matrix Metalloprotease -2 (Metalloprotease). The sequence sets were composed of cleaved and uncleaved sequences identified in experiments or generated by examining naturally occurring targets (and non-targets) of each protease (see Materials and Methods). We preferentially chose datasets in which 
cleaved and uncleaved sequences were identified in the same experiment. For HCV NS3/4 protease, HIV Protease 1 and Granzyme B, we were able to identify experiment-derived datasets $(22,29,30)$. For TEV protease and MMP2 protease, we were able to obtain experimentally cleaved datasets(31-33) but uncleaved sequences were not available. Therefore, we generated a synthetic dataset of uncleaved sequences using a two-residue protein walk approach, utilized in previous computational and experimental work $(22,29)$, in which if all cleavage sites of a protease in a given protein (or viral polyprotein) are known, it can be inferred that the remaining epitopes in the protein (polyprotein) are uncleaved . It is possible that these synthetically generated uncleaved sequences may include a small number of cleaved sequences. However, experimental results from Shiryaev et al (29) suggest that misclassification of uncleaved sequences obtained using this approach is low. Therefore, in the absence of a directly experimentally determined uncleaved dataset for TEV protease and MMP2, we utilized this previously developed approach for uncleaved dataset creation.

\section{Developing an energetic discriminatory scoring function based on structural simulations:}

We hypothesized that determinants of substrate cleavage by a protease include (a) protease-peptide interfacial interaction energy including electrostatics, (b) the adoption of a catalytically competent conformation of the protease active site machinery in the bound state (near-attack conformation), and (c) a reorganization penalty that captures the propensity of a given substrate to adopt the extended conformation required for positioning the scissile bond in a cleavage-prone location in the protease active site. We 
created atomic resolution models for each peptide-protease complex and computed each of these terms as described below.

To model the conformation of each substrate peptide complexed with the active conformation of the protease, we created atomic resolution models within the context of the Rosetta macromolecular modeling software. Each known peptide substrate was threaded on the respective modeled near-attack conformation generated from the protease crystal structures (Figure 1D), and the resulting complex was allowed to computationally relax into a local energy minimum using Rosetta FastRelax(34), followed by scoring this modeled conformation using Rosetta and Amber's MMPBSA modules (Fig. 1E).

In addition to the interaction energy evaluated using Rosetta (Fig 1F), which includes a Coulombic model of electrostatics (called fa_elec, weighted at $\sim 0.25$ in the Rosetta energy function), we also evaluated binding electrostatics by using Amber's MMPBSA module (Fig. 1G). The MMPBSA approach is based on solving the Poisson-Boltzmann equation, which is a more accurate model of the electrostatic interactions than the Coulombic one. While this approach of combining Rosetta and Amber electrostatics entails double-counting, we reasoned that the Rosetta energy function has been weight optimized for all of its component terms simultaneously including fa_elec. Thus, we decided to include fa_elec even upon inclusion of the AMBER electrostatics score. In tests on four protease systems, we found that the discriminatory capability of the score function was not affected when the Rosetta energy term fa_elec was excluded. We included two other terms in our discriminator scoring function: First, we included a term ("reorganization penalty") that 
captures the propensity of a given substrate to adopt the extended conformation observed in crystal structures of all proteases (Fig. 1H). Second, the deviation of the active site from ideal catalytic geometry (a pseudo-energy term) upon energy minimization (Fig. 1I), which captures the fit of a given substrate to the catalytically competent conformation of the protease, was included. These scores - energetic descriptors of the peptide-protease complex in a near-attack conformation - were combined using a linear weighting approach to obtain a discriminatory score function such that lower scores are predicted to energetically fit better in the active site (Figure 1F-I).

\section{Recapitulation of known protease specificity profiles:}

Each predicted substrate binding set for each protease consists of a large set of evaluated peptide sequences, atomic-resolution bound structures, and predicted binding energies of individual peptides to the near-attack state of the enzyme. We compared our predictions with experimentally determined specificity data from peptide library screening. Briefly, in these experiments, peptide (or peptide-cDNA fusion) libraries are generated and treated with protease of interest, cleaved and uncleaved populations of peptides are captured and identified using (deep) sequencing or mass spectrometry, and cleavage probability is assigned using Enrichment of a given peptide sequence in the cleaved population versus the uncleaved.

We found that for each of the five proteases, the distribution of discriminator scores was bimodal and cleaved and uncleaved sequences were separated in a statistically significant manner ( $p$-values calculated using the Wilcoxon rank test; Fig. 2A-E). To quantify the 
performance of the discriminator in the task of separating cleaved from uncleaved substrates, we performed a score threshold-based binary classification of the sequences into cleaved and uncleaved sets and calculated the area under the resulting receiveroperator curve (auROC; perfect discrimination would yield an auROC of 1.0; the expected auROC for a random ordering of the peptides is 0.5). The auROC values for the five proteases ranged between ( 0.86 for MMP-2 to 0.98 for TEV-PR), demonstrating robust discrimination using energetics (Figure 2G). The critical point of the auROC plot represents the optimal tradeoff between false positive and false negative rates. We found that false positive rates at critical points ranged from 0.04 (TEV-PR) to 0.24 (MMP-2), suggesting robust discrimination of the substrates into cleaved and uncleaved sets with a small but significant false positive rate (Table S1). We note that weights used for combining the five score terms were initially optimized to maximize discrimination for HCV NS3/4 protease (five weight terms over approximately 2100 data points), yet TEV-PR displays the best performance in terms of both auROC and critical point values using this weight set. These results demonstrate the generality and robustness of the energy-based scoring function.

To evaluate the ability of the discriminator to identify cleaved sequences from the entire pool of sequences - a task that would aid in novel substrate identification - we calculated the fraction of truly cleaved sequences in the top-scoring $N_{\text {cleaved }}$ sequences, where $N_{\text {cleaved }}$ is the number of cleaved sequences in the dataset. This Enrichment value is compared to background Enrichment, i.e. fraction of cleaved sequences in the dataset (reflecting a scenario when the ranking is performed by randomly shuffling the list of sequences). We find that in all cases a significantly higher fraction of sequences was enriched compared to 
the background with Enrichment ratios ranging from 3-fold (HIV-PR) to 19-fold (TEV-PR) (Figure 2F). We compared the Enrichment obtained using our discriminator with that obtained using SitePrediction (26)- a sequence-based machine learning method that relies on training with experimental data. For each protease, we trained a SitePrediction model with randomly chosen $20 \%$ of the known cleaved sequences and used the remaining dataset for testing. For all proteases, we find that our unbiased, biophysics-based approach yielded similar or higher Enrichment values as SitePrediction models trained separately on each individual protease. The lack of training on known experimental data makes the structure-based discriminator more widely applicable.

\section{Optimization of scoring and sampling approaches:}

To investigate the contribution of each score term to the discriminator scoring function, we evaluated the discrimination performance of various score term combinations. We found that while the majority of the discriminatory power could be attributed to Rosetta interface residue energies, all five terms do contribute to the observed prediction metrics when they are serially included along with the Rosetta energy. While the increases in auROC compared to Rosetta energies-only scoring functions were modest, Enrichment values benefited significantly by the inclusion of the additional terms, e.g., for Granzyme B inclusion of the AMBER electrostatics score and secondary structure propensity increases Enrichment from 0.70 to 0.87 (Supplementary Fig S1, Table S2). As auROC measures the overall difference in the two distributions (cleaved and uncleaved), and Enrichment measures the rank ordering of sequences, we conclude that inclusion of additional terms 
serves to subtly alter the calculated energy landscape and "rescue" some false negatives (cleaved sequences that score comparably to low-energy uncleaved ones).

We next investigated whether optimization of weights of the energetic scoring terms could improve performance. We used a grid-based optimization scheme in weight space to maximize Enrichment. While keeping Rosetta protease energy fixed, we optimized four free parameters by enumerating all combinations of peptide residue energy (0.3-1.3 in increments of 0.1 , constraints $(2.5-3.5$ in increments of 0.1$)$, secondary structure $(0.005-$ 0.02 in increments of 0.005), and electrostatics (0.1-0.3 in increments of 0.05). The ranges were chosen after a coarse-grained parameter sweep to find good starting parameters, and by considering the orders of magnitudes of raw scores of the score terms. For example, the raw score for the Secondary Structure Propensity term ranges between 0-200 (number of fragments from the top 200 that have an RMSD greater than 3.0 A compared to the crystallographic conformation of the peptide). As the Rosetta residue energy weight was 1 , we explored weight ranges of $0.005-0.02$ for the secondary structure term. The results of this optimization are listed in Table S3.

We next examined the impact of sampling flexibility of the backbone and sidechain degrees of freedom at the protease-peptide interface (Supplementary Figure S2) and found that limiting the backbone degrees of freedom of the protease, while sampling the full backbone DOFs of the peptide, yielded the highest Enrichment values. Previous studies with farnesyltransferase enzyme similarly observed that greater sampling of the peptide degrees of freedom increased performance (35). When the protease backbone was allowed 
to move in an unconstrained manner, several uncleaved sequences adopted energetically favorable conformations. While some of these false positives can be attributed to limitations of the simulation force fields and sampling strategies, these results indicate that side chain flexibility in the protease pockets coupled to peptide backbone flexibility are key contributors to the molecular recognition observed at these interfaces.

Finally, we explored the contribution of maintaining, during each simulation, the scissile peptide bond in a near-attack conformation with respect to the protease catalytic machinery using geometric constraints, by performing simulations without these geometric constraints, and/or removing the constraint scores from the discriminator scoring function. In each case, a decrease in Enrichment was observed (Supplementary Figure S3), providing further support for our rationale that specificity in protease-peptide molecular recognition is not simply a ground state binding phenomenon, but is contingent upon the relative energetics of the near attack substrate conformation during the nucleophilic attack step.

\section{Combining sequence and energetic signatures using machine learning leads to higher discriminatory power}

Current approaches for protease specificity prediction, including the SitePrediction tool discussed above, PCSS server (22) and PROSPER(25), use machine learning of sequence patterns in known experimental data. To more extensively compare our structure-based specificity prediction with current sequence-based approaches we trained support vector machines (SVMs) with sequence-only, energetic-only and both sequence and energetic 
features (Methods). For the energy-based SVM, the (unweighted) energy terms described above were treated as features ("interface protease residue energy", "interface peptide residue energy", "constraints energy", "reorganization penalty" and "MMPBSA electrostatic binding energy"), whereas sequence-based features were generated using a protocol described by Barkan et al.(22). We found robust discrimination of the substrate sequences using energy-based SVMs trained individually on each protease in 5-fold cross-validation test (Fig. 3A). The values of auROC obtained using these SVMs are higher than those obtained with scoring using a linear weighting scheme (Fig. 3B, black and gray bars), due likely to the use of a non-linear kernel function and training on individual datasets. When compared to a purely sequence-based SVM, the energy-based SVM consistently leads to higher auROC values for all datasets, and an SVM constructed based on sequence and energy features displays a high AUC value when compared to solely sequence-based and energy-based based SVMs (Fig 3B). These results indicate that structural/energetic features contribute information that is orthogonal to that obtained from sequence-only features.

To ensure that the increased discriminatory ability observed upon combining sequenceand energy-based features is not a result of data over-fitting, we performed a crossvalidation procedure where in-set training (classification) and out-of-set testing (generalization) was performed by randomly splitting the datasets into training and test subsets (36). We find that the performance of the method as indicated by the accuracy of prediction, does not appreciably alter when a significantly smaller training dataset is used for the energy-based SVMs, and the classification and generalization performance converge 
as the training set size increases (Fig. 3C-E, Supplementary Figure S4). The convergence between classification and generalization occurs at higher training set fraction for the sequence-based SVMs than energy-based ones, demonstrating that the key energetic signatures underlying discrimination can be captured with a smaller dataset compared to the corresponding sequence signatures (Supplementary Figure S4). Thus, energetic feature-based SVMs can outperform sequence-based ones, and the two sets of features can be combined to obtain more accurate classification than either set of features independently.

\section{Multi-body interaction networks at the interface underlie improved discrimination}

To investigate the underlying reasons for the observed increase in prediction efficiency when structural features are used, we identified several peptide sequences that are consistently misclassified by the sequence-based approach but are correctly classified by the structure-based approach. In several cases, we find that the increased classification ability could be attributed to interaction networks composed of multiple substrate and protease residues. A sequence-only approach would require a significantly larger training data than a relatively unbiased energy-based approach to directly "learn" multi-body correlations (interactions).

Three examples of these interaction networks are described below: 
1. The structure-based discriminator can identify context-dependence of the substrate residue interactions more readily than a sequence-based approach, especially in cases where sequence preference at a given substrate site is not pronounced. For example, for the HIV protease, cleavage occurs between small non-polar amino acids and sequence preference at any other site is not particularly pronounced. As a result, GPGTASRP (Fig 4A) is misclassified as "cleaved" by the sequence-based SVM for HIV protease-1. There are no pronounced sequence preferences at position P3' (Supplementary Figure S1D). The structural model of this sequence, however, shows that the guanidinium group of the arginine sidechain (P3') is packed in the vicinity of R8, a key residue, whose interaction with D29 is critical for HIV protease structural (dimer) stability (37). Thus, the presence of an arginine at this P3' position would lead to lack of cleavage of the substrate, unless a secondary interaction relieves the electrostatic repulsion between the substrate arginine sidechain and the guanidinium group of R8. The subtle balance of these protease-substrate interactions can be captured by the electrostatics calculations in our approach.

2. The energy-based discriminator is able to detect hydrogen bond networks between substrate residues, including those mediated by the protease structure. For example, for the sequence SQAYPIVQ (Fig. 4B), the sidechain of the tyrosine residue at position P1 forms a hydrogen bond network with the P4 position on the substrate and the R8 of the protease chain. This likely allows the protease to recognize and cleave this substrate.

3. Another set of interactions that our structural approach correctly characterizes are those mediated by proline and glycine residues, as these have specific backbone preferences that 
can affect the peptide backbone conformation. Figure 4C is an example of a sequence, KPAIIPDR, which is experimentally shown to be uncleaved by the HCV NS3 protease. The sequence-only approach misclassifies this sequence as cleaved, likely on account of the non-polar isoleucine residues at the P1, P1' residues. However, the proline residues present at P5 and P1 substrate positions bend the substrate backbone into a conformation that results in the disruption of the stabilizing backbone hydrogen bond network, which drives the extended substrate conformation optimal for cleavage. The Rosetta energy function detects the disruption of this backbone hydrogen bond network, and thus the energy-based approach correctly classifies this sequence as 'uncleaved'.

\section{Discovering novel sequence specificities HCV NS3 4A Protease}

To further investigate the predictive ability of the energetic-discriminator in a blind test, we used our simulations to identify novel cleaved substrates for the HCV NS3/4 protease. The residue identities on the substrate peptide at positions P6 through P2 were sampled and scored as described in Methods using the structure-based discriminator. A total of 26,400 candidate sequences were evaluated (out of the possible $20^{5}=3.2$ million) in a twostep procedure of sequence sampling as described in Methods, low-scoring sequences were clustered and were further pruned to identify sequence motifs that were novel (i.e., absent from the dataset used for developing the discriminator). We identified four such sequence motifs (Fig. 5A), whose scores overlapped with the distribution of scores obtained from known cleaved sets. At least one peptide sequence was selected from three of the four identified motifs, and these were tested experimentally using a Yeast Endoplasmic Reticulum Sequestration Screen (YESS system) based assay $(28,38)$ (Fig. 5B). 
In this assay, the protease and substrate are co-expressed in active forms in the ER of yeast, and the substrate is targeted to the cell surface by fusion to the cell surface protein Aga2p. Proteolysis is detected using fluorescent antibodies against the HA and FLAG tags that flank the substrate. We confirmed that the cleavage of the wild type substrate sequence (DEMEECA- canonical HCV NS3 cleavage sequence present between NS4A/4B on the polyprotein) results in the detachment of the FLAG tag from the AGA2 surface-signaling moiety, thus resulting in a FLAG:HA ratio of zero for complete cleavage and a ratio of one for no cleavage when an inactive variant of the protease (S139A) is used (Fig. 5 C). Several previous studies $(29,39,40)$ have shown that the HCV protease cleaves between C/S or C/A residues (P1/P1') - however, the specificity at other positions can be broad and has not been explored fully. In all our predicted substrates (that we tested experimentally) the $\mathrm{P} 1 / \mathrm{P} 1$ ' positions are still maintained as the known canonical sequence $\mathrm{C} / \mathrm{S}$, and our goal was prediction of different P6-P2 patterns. We, therefore, reasoned that the cleavage position of our substrates would not be altered as they retain the canonical P1/P1' cleavage pattern. The FLAG and HA signals were detected using flow cytometry. The observed FLAG/HA ratios (Fig. 5 C,D) demonstrate that three out of four predicted sequences showed cleavage with ratios $<0.5$, whereas control assays with the $\mathrm{S} 139 \mathrm{~A}$ inactive protease variant showed significantly higher $(>0.85)$ ratio, demonstrating that the observed cleavage is not due to a non-specific endogenous yeast enzyme.

Out of the four sequences that are predicted as cleaved, one sequence - CEDYFCSG - shows a high FLAG/HA ratio, and represents a prediction failure. These results are consistent with 
the $\sim 75 \%$ True Positive and $\sim 25 \%$ False Positive rates (Fig. 2F) observed in the performance of the discriminator on known cleaved and uncleaved datasets, i.e., approximately one out of four sequences identified is expected to be a false positive sequence. We also identified two predicted uncleaved substrates, and these show lack of cleavage when co-expressed with either wild type protease or the inactive protease variant, as expected. The FLAG:HA ratios for the novel identified substrates are higher than positive control LY104, indicating that the substrates identified are suboptimal. However, our test for novel substrates is particularly stringent as we chose sequence motifs that have previously not been identified in multiple studies of HCV NS3/4 protease. Thus, the developed discriminative score function and validating assay provide a method to screen for potential novel biological targets of this viral protease that is also a drug target.

\section{Discussion:}

Proteolytic cleavage is a key component of diverse and ubiquitous biological processes such as apoptosis, blood clotting, viral maturation, and cancer(41). Developing a generalizable, predictive model for protease specificity would enable identification of potential novel substrates for furthering our understanding of protease biology and enhancing our ability to design inhibitor small molecules to chosen proteases. We developed a structure-based approach for specificity prediction using Rosetta and Amber force fields that provides atomic resolution insights into the molecular recognition at protease-substrate interfaces. We found that structural models robustly recapitulate known protease specificities for each of the four major protease classes (serine, cysteine, 
aspartic, and metallo-proteases) with little training on experimental data, and in several cross-validation tests. When combined with a machine learning algorithm our energybased approach outperforms current bioinformatics-based approaches (24) on benchmark sets, and a further increase in discrimination is achieved when both structure-based and sequence-based approaches are combined. To further test the utility of our approach in a blind manner, we used it to predict four novel substrate sequences for HCV NS3/4 protease, tested these predictions experimentally, and found that three of the four novel predicted cleaved sequences were cleaved by the protease; a success rate similar to the benchmark set was achieved in the blind experimental test.

The value of using energetic information in the discriminator is evident in the protease structure-dependent interaction networks that are captured in the energetic signatures. These interaction networks are equivalent to pairwise and multi-body correlations in the sequence data. Given 20 amino acid types at every substrate peptide position, a relatively large number of training sequences are required to "learn" pairwise and higher-order correlations between positions, whereas only $\sim 2000$ sequences (among them $\sim 200$ cleaved) are available in the experimental benchmark datasets. The structure-guided, energy-based discriminator has the advantage of being generalizable, relatively unbiased and is able to recapitulate key interactions that stabilize the peptidase - peptide interface as well as predict novel interactions not present in the training data. Success in using structure-based energetic signatures and molecular docking for binding partner identification has been achieved for several peptide recognition modules such as $\mathrm{SH} 3$ and PDZ domains(42-46), major histocompatibility complex (47) and for the enzymes 
methyltransferase(48), farnesyltransferase (35), and $\operatorname{HIV}$ protease $(49,50)$. We show here that a structure-based approach, guided by the knowledge of mechanism, can be successfully integrated with machine learning to predict substrates for a mechanistically diverse enzyme family such as proteases with high accuracy.

Proteolytic sites in full-length proteins are more often found in exposed regions of the structure, and more frequently in flexible loops and beta conformations compared to buried regions and alpha helices (20). A substrate sequence generally adopts an extended conformation in the protease active site (4), and surface-exposed loops and beta-strand regions are likely to pay a smaller reorganization penalty to adopt this extended conformation. Therefore, we incorporated the local structure preferences of the substrates in our datasets by computing local sequence-structure compatibility - an implicit assumption in our approach is that every candidate peptide sequence is equally accessible to the protease active site. This assumption is valid when analyzing the extended substrate specificity of the protease, but for the task of predicting cleavage sites in a given whole protein sequence, additional solvent accessibility and structural information are expected to modulate cleavability. Barkan et al. have shown that incorporation of such features improved prediction of cleavage sites in whole protein sequences. Furthermore, Julien et al. (8) found that cleavage efficiencies of protein substrates identified using a high throughput mass spectrometry-based approach and their synthetic peptide counterparts were correlated. Taken together, it appears likely that local primary sequence specificity (modeled here) largely determines the identity of cleavage sites, although the context of the cleavage site modulates the kinetics of cleavage. 
Comparing the performance of the discriminator for the different protease systems included in the benchmark set highlights the strengths and limitations of our approach. Highest Enrichment of cleaved sequences in the top-ranked population is observed for TEV and Granzyme B proteases (Fig. 2), where the active site is relatively rigid and steric effects and hydrogen bond interactions are the major contributors to specificity, highlighting the strength of the Rosetta force field in modeling these effects. However, performance is more modest for the metalloenzyme MMP-2, which features a zinc ion in the active site, and for the HIV protease, in which loop residues mediate molecular recognition. For these systems, inaccuracies in the modeling of flexibility of the active site conformation, and lack of explicit consideration of entropy changes can lead to increased misclassification. More exhaustive sampling of the backbone degrees of freedom of the loop structural elements is likely to improve performance as observed in other studies of peptide-protein molecular recognition $(35,51)$. Finally, while modeling catalytic residue conformations using geometric constraints appears to be a reasonable approximation for most systems considered here as evidenced by success in discrimination, electronic effects may be involved in the vicinity of the active site, especially for the metalloenzyme MMP-2. We also investigated alternative protonation states of key catalytic residues (nucleophiles serine, cysteine, hydroxyl and bases histidine, aspartic acid) in the MM-PBSA pipeline, but these charge changes did not lead to any appreciable increase in the performance (data not shown). It is likely that quantum mechanical (QM) calculations may be required to model these effects more accurately. However, the high computational cost of detailed QM simulations precludes the use of such calculations for the thousands of substrate-enzyme 
pairs considered in our study. Advances in QM simulation methodology(52) and computational infrastructure are likely to bridge this gap in the future.

In contrast with sequence-based specificity prediction approaches, the unbiased nature of the biophysical substrate specificity predictor developed here should allow the modeling of specificity of protease variants for which experimental data are not available, such as newly emerged drug-resistant variants(53) of viral proteases as well as newly-discovered and/or uncharacterized proteases, whose sequences are homologous to proteases of known structure. Energy-based specificity prediction will also aid in the design of protease variants targeted to specific substrates. Current approaches for protease design rely on library-based screening/selection(28, 54, 32) in vivo. These directed evolutionary trajectories often proceed via incremental "generalist"(55) intermediates that display relaxed specificity, and are, therefore, toxic to cells (or the proteases undergo self-cleavage) and are never identified in the selection. A structure-guided computational design approach based on the evaluation of interaction energies of substrates with protease variants should allow for multiple simultaneous substitutions ("jumps" in the sequence landscape) to allow specificity switching without generating generalist toxic intermediates. Combining structural computation using the discriminator described here with directed evolution should enable more efficient protease specificity design.

\section{Materials and Methods \\ Curation of Benchmark Datasets}


Each protease used in the study exhibits diverse mechanisms of action, interface recognition modes, varied folds and biological functions (Figure 6) - e.g. TEV Protease (cysteine proteases), HCV NS3 protease (serine proteases), Granzyme B (serine protease), HIV Protease-1 (aspartyl protease) and Matrix Metalloprotease -2 (Metalloprotease). The sequences of cleaved and uncleaved substrate peptides for each protease were obtained as detailed below:

HCV protease: We obtained the cleaved and uncleaved sequence sets from a deep sequencing study by Shiryaev et al (29). Only sequences with signals above a threshold (Zscore value> 3) at all three time points in their study were considered in order to avoid noise from deep sequencing analyses. We also incorporated sequences from a study by Rögnvaldsson et al(56). Merging both individual sets generated a set with 196 cleaved and 1943 uncleaved sequences.

HIV-PR: 374 cleaved and 1251 uncleaved sequences were obtained from Rögnvaldsson et $\operatorname{al}(56)$.

TEV protease: The cleaved set of 68 sequences was curated from results obtained by Kostallas et al. (31) and Boulware et al. (32). Due to the absence of a large uncleaved sequence dataset for the TEV protease, we synthetically generated the uncleaved dataset using a two-residue walk on the TEV polyprotein sequence. The TEV protease is expected to cleave only at one specific site in the polyprotein. Half of the sequences were randomly discarded to generate a dataset of 1520 uncleaved sequences. We ensured that the 
sequence distribution was not biased toward any specific amino acid type at any peptide position (Figure 6).

Granzyme B: The cleaved sequence set was obtained and uncleaved sequence set was adapted from Barkan et al. (22). A subset of the uncleaved sequences was randomly chosen and the amino acid identity at P1 was randomly mutated to all amino acid identities except aspartate and glutamate. A total of 353 cleaved and 1973 uncleaved sequences were chosen.

Matrix Metalloprotease: The cleaved sequence set of 455 sequences was obtained from Ratnikov et al (57). To curate the uncleaved sequence set, we scanned the CutDB(58) database for MMP-2 protein substrates. Excluding the known cut sites in these proteins, the rest of the protein sequence was treated as uncleaved using a two-residue walk to generate an uncleaved sequence set of 1818 sequences for MMP-2.

The sequence sets are provided in Supplementary Information.

\section{Starting model generation for simulations:}

We constructed models of peptide-protease bound complexes using high-resolution crystal structures culled from the Protein Data Bank (PDB) (Table 1)(59-63). Crystal structures were filtered based on the following criteria: a resolution lower than $2.6 \AA$ and a peptide or peptidomimetic inhibitor bound in the crystal structure. We remodeled the crystallographic conformation of the bound peptide to mimic the near-attack conformation 
for nucleophilic addition step of the proteolysis reaction by enforcing catalytic geometries obtained from mechanistic quantum mechanics simulations and/or crystal structures of proteases bound to inhibitors during Rosetta FastRelax simulations. The selected crystal structures were optimized using a Rosetta FastRelax protocol to find a low energy, stable structure, which was used as a starting point in further calculations. Constraints were applied during FastRelax in order to maintain active site geometry and keep the protease in a catalytically active conformation. Co-ordinate constraints were also applied to the protease backbone to ensure that the structure does not drift away from the crystallographic conformation, while still minimizing energy, as previously described (64).

\section{Calculating Rosetta and Amber energies:}

Starting from the relaxed crystal structure described above, we threaded the candidate peptide sequences to generate models of the protease-peptide complex corresponding to each sequence. The energy of the resulting conformation was minimized with constraints using Rosetta FastRelax and ten models were generated for each sequence. During this protocol, the protease backbone was constrained, protease side chains were allowed complete conformational flexibility, whereas peptide side chains and backbone were allowed to sample all degrees of freedom including backbone, sidechain and rigid body orientation with respect to the protease. The side chains of the catalytically active residues were constrained with respect to the scissile peptide bond of the substrate using enzyme design-style Rosetta constraints. This model represents a pre-transition state near-attack conformation for each of the peptide substrates for the protease. The resulting models were scored with Rosetta's Talaris2013 energy function. 
Total residue energies for protease interface residues were extracted for all ten structures representing a single sequence, averaged and stored as "protease energy". Interface residues were defined as those whose C-alpha atom was within $8 \AA$ of any peptide residue's C-alpha atom. We experimented with 8,10 , and $12 \AA$ as the cutoff distance for defining the protease shell, but we found that the discriminator performance was robust to this cutoff value. The sum of total residue energies over all peptide residues was averaged and stored as "peptide energy". Total interface energy was defined as the sum of protease and peptide energies. These models were also scored for "constraint energy" based on the deviation of active site residues geometries from idealized ones. Each energy term was used as a feature during machine learning (see below).

Sampling of the peptide backbone and protease and peptide side chains degrees of freedom was performed before calculating scores for a given complex structure. We optimized the structure sampling protocol by investigating several combinations of sidechain and backbone flexibility for the peptide and the protease, and their relative rigid-body transform. Allowing peptide backbone and sidechain flexibility, and protease sidechain flexibility afforded the highest discriminatory capability (Supplementary Figure S2). All calculations were performed with the interface RosettaScripts $(65,66)$. Sample $\mathrm{xml}$ files used can be found in Supplementary Information. The AMBER Tools 12 MMPBSA(67) application was used to calculate the electrostatic contribution to the bound state energy over the unbound energy for the protease-peptide complex. Run scripts are provided in Supplementary Information. 


\section{Local sequence-structure compatibility}

Rosetta's FragmentPicker (68) Tool was used to analyze the propensity of a peptide sequence to adopt an extended conformation that is found in protease active sites. We picked 200 fragments for a given peptide sequence, and calculated the RMSD of each fragment with the bound conformation of the peptide. The number of fragments with RMSD $>2.0$ in the set of 200 top fragments compared to the bound conformation was used as the score.

\section{Support Vector Machines}

An SVM constructs a hyper plane between two sets of data points in multi-dimensional "feature" space, based on a predefined kernel function in order to maximally separate the two datasets. We used the built-in SVM function (MATLAB 2015) with a radial-based kernel function following Barkan et al. (22). In the RBF kernel, parameters $C$ and $\gamma$ need to be adjusted: $C$, also called cost factor, is a regularization parameter that controls the trade-off between maximizing the margin and minimizing the prediction error, while $\gamma$ is a kerneltype parameter that dominates the generalization ability of SVM by regulating the amplitude of the kernel function. We optimized the training parameters of SVM based on 5cross-validation tests. $C$ - and $\gamma$-values of 10 and 10 , respectively, were used.

Sequence features: Each position within the sequence was considered to be one feature. The one letter amino acid codes were transformed into an index, which was calculated 
from the rank of the amino acid residue in an alphabetical ordering of all amino acids as well as on its position in the sequence from $\mathrm{N}$ to $\mathrm{C}$ terminus on the substrate chain as in Barkan et al.(22). All 20 amino acids at each position in the peptide were assigned a number using the formula $\mathrm{n} * 20+\mathrm{i}$, where $\mathrm{n}$ represents the position of the residue in the peptide sequence and i represents the position of the residue in an alphabetical ordering of amino acids by their one letter code.

Structure features: Each contributing discriminator energy score was imported into the SVM as an independent feature. The structure-based Rosetta energies ("Interface residue peptide energy", "Interface residue protease energy", "Reorganization penalty", "constraint energy") and Amber energy ("electrostatic energy") were used as features. The SVMs were cross-validated using an 80-20 bootstrap over 1000 iterations.

\section{Generation of a computational library for HCV NS3/4A substrate from P6 through P2 positions:}

The mutational scanning was executed in two parts. We generated models of the proteasepeptide complex for substrate positions P6 through P4, energy minimized and scored them using the computational protocol descried above. Ten structures were generated for each sequence. The models were evaluated using the weighted optimized energies as used in the discriminator. The top scoring 66 sequences were identified, and 26,400 models were generated by sampling P3 and P2 substrate positions for each sequence. These 26,400 models were subjected to energy minimization and score calculations as previously described. To calculate their final score, Rosetta interface energy, constraint energy, and 
AMBER MMPBSA electrostatic energy were used at the optimized Enrichment values. To reduce computational costs, the reorganization penalty score was not included in the final score calculation since it did not measurably change the auROC value in the benchmark set (Supplementary Fig S1). The sequences that lay in the score distribution of the native cleaved sequences were further analyzed. These were filtered to be most different from the initial HCV cleaved sequence distribution and clustered using Hamming distance into 4 main sequence pools- CED*, LEE*, FED*, YED*. Representative sequences from the first three sequence clusters were tested experimentally.

\section{Flow Cytometry:}

We used the Yeast ER Sequestration and Screening Assay (YESS) for in vivo testing of predicted substrates of the HCV protease. The LY104 construct for the assay was a gift from Y. Li, B. Iverson, and G. Georgiou (University of Texas at Austin). The sequences to be tested were cloned into LY104 using a Restriction Free Cloning method(69). Table S4 lists all the primers associated with the cloning protocol.

The positive control and test plasmids were then transformed into the EBY100 competent yeast strain. They were plated on selective complete (SC) media ( $20 \mathrm{~g} / \mathrm{L}$ glucose) with a selective amino acid mix ( -Trp, - Ura). After two days of growth, a single colony was transferred to a $2 \mathrm{~mL} \mathrm{SC}$ media culture tube supplemented with $2 \mu \mathrm{L}$ of $1000 \mathrm{x}$ antibiotics (carbenicillin, kanamycin). The growth cultures were incubated for $\sim 24 \mathrm{~h}\left(\mathrm{OD}_{600} 2.0-3.0\right)$ in a $30{ }^{\circ} \mathrm{C}$ shaking incubator. $1.5 \times 10^{7}$ cells $\left(\mathrm{OD}_{600} \sim 0.5\right)$ were pelleted and resuspended in 2 $\mathrm{mL}$ induction media ( $20 \mathrm{~g} / \mathrm{L}$ galactose, $2 \mathrm{~g} / \mathrm{L}$ glucose) supplemented with $2 \mu \mathrm{L}$ each of 
1000x antibiotics (carbenicillin, kanamycin). The induction cultures were grown overnight at $30{ }^{\circ} \mathrm{C}$ to an $\mathrm{OD}_{600}$ of 1-1.5. All spins in the protocol were done at 3000 r.c.f for 5 min. The induced cultures were pelleted and washed with $500 \mu \mathrm{L}$ PBS followed by $500 \mu \mathrm{L}$ PBS+ 0.5\% BSA. $1 \mu \mathrm{L}$ of each antibody stain(anti-FLAG, anti-HA) was incubated with $10^{7}$ cells for 30 min at $4{ }^{\circ} \mathrm{C}$. The samples were resuspended by vortexing and incubated at RT for an additional 30 min. The cells were washed with $100 \mu \mathrm{L}$ PBS with $0.5 \%$ BSA, pelleted and then resuspended in $500 \mu \mathrm{L}$ PBS. Samples were diluted to achieve a final concentration of $10^{6}$ cells/mL and then FITC (anti-HA) and PE(anti-FLAG) intensities were detected using a Flow Cytometer (Beckman Coulter Gallios).

\section{Acknowledgements}

This work was supported by the National Science Foundation grant NSF-MCB 1330760. ABR was supported by the National Science Foundation Graduate Research Fellowship under Grant No. NSF DGE-1433187. We thank D. Case, J. Swails and P. Janowski for assistance with AMBER MMPBSA. We thank T. Rögnvaldsson for providing us with cleavage data for HCV NS3 Protease and HIVPR-1, A. Sali and D. Barkan for providing the Granzyme B dataset, and Y. Li, B. Iverson, G. Georgiou for providing the LY104 plasmid used in the YESS assay experiments.

\section{References:}

1. López-Otín C, Bond JS (2008) Proteases: multifunctional enzymes in life and disease. J Biol Chem 283(45):30433-7. 
2. Hedstrom L (2002) Introduction: Proteases.

3. Hedstrom L (2002) Serine Protease Mechanism and Specificity. Chem Rev 102(12):4501-4524.

4. Tyndall JDA, Nall T, Fairlie DP (2005) Proteases universally recognize beta strands in their active sites. Chem Rev 105(3):973-99.

5. Powers JC, et al. (1993) Proteases--structures, mechanism and inhibitors. Agents Actions Suppl 42:3-18.

6. Rawlings ND, Salvesen G (2013) Handbook of Proteolytic Enzymes. Handbook of Proteolytic Enzymes doi:10.1016/B978-0-12-382219-2.00848-6.

7. Rawlings ND, Barrett AJ, Bateman A (2010) MEROPS: the peptidase database. Nucleic Acids Res 38(Database issue):D227-33.

8. Julien 0, et al. (2016) Quantitative MS-based enzymology of caspases reveals distinct protein substrate specificities, hierarchies, and cellular roles. Proc Natl Acad Sci U S A 113(14):E2001-10.

9. Di Cera E, Cantwell AM (2001) Determinants of thrombin specificity. Ann N Y Acad Sci 936:133-46.

10. Scheel TKH, Rice CM (2013) Understanding the hepatitis C virus life cycle paves the way for highly effective therapies. Nat Med 19(7):837-849.

11. Drag M, Salvesen GS (2010) Emerging principles in protease-based drug discovery. Nat Rev Drug Discov 9(9):690-701.

12. Eder J, Hommel U, Cumin F, Martoglio B, Gerhartz B (2007) Aspartic proteases in drug discovery. Curr Pharm Des 13(3):271-85.

13. Tawfik DS (2014) Accuracy-rate tradeoffs: how do enzymes meet demands of 
selectivity and catalytic efficiency? Curr Opin Chem Biol 21:73-80.

14. Poreba M, Drag M (2010) Current strategies for probing substrate specificity of proteases. Curr Med Chem 17(33):3968-95.

15. Turk BE, Huang LL, Piro ET, Cantley LC (2001) Determination of protease cleavage site motifs using mixture-based oriented peptide libraries. Nat Biotechnol 19(7):6617.

16. Backes BJ, Harris JL, Leonetti F, Craik CS, Ellman JA (2000) Synthesis of positionalscanning libraries of fluorogenic peptide substrates to define the extended substrate specificity of plasmin and thrombin. Nat Biotechnol 18(2):187-93.

17. Fretwell JF, K Ismail SM, Cummings JM, Selby TL (2008) Characterization of a randomized FRET library for protease specificity determination. Mol Biosyst $4(8): 862-70$.

18. van den Berg BHJ, Tholey A (2012) Mass spectrometry-based proteomics strategies for protease cleavage site identification. Proteomics 12(4-5):516-29.

19. Ratnikov B, Cieplak P, Smith JW (2009) High throughput substrate phage display for protease profiling. Methods Mol Biol 539:93-114.

20. Agard NJ, et al. (2012) Global kinetic analysis of proteolysis via quantitative targeted proteomics. Proc Natl Acad Sci U S A 109(6):1913-8.

21. Vizovišek M, Vidmar R, Fonović M, Turk B (2016) Current trends and challenges in proteomic identification of protease substrates. Biochimie 122:77-87.

22. Barkan DT, et al. (2010) Prediction of protease substrates using sequence and structure features. Bioinformatics 26(14):1714-22.

23. Boyd SE, Garcia de la Banda M, Pike RN, Whisstock JC, Rudy GB (2004) PoPS: a 
computational tool for modeling and predicting protease specificity. Proc IEEE Comput Syst Bioinform Conf:372-81.

24. Song J, et al. (2011) Bioinformatic approaches for predicting substrates of proteases. J Bioinform Comput Biol 9(1):149-78.

25. Song J, et al. (2012) PROSPER: an integrated feature-based tool for predicting protease substrate cleavage sites. PLoS One 7(11):e50300.

26. Verspurten J, Gevaert K, Declercq W, Vandenabeele P (2009) SitePredicting the cleavage of proteinase substrates. Trends Biochem Sci 34(7):319-23.

27. Li B-Q, Cai Y-D, Feng K-Y, Zhao G-J (2012) Prediction of protein cleavage site with feature selection by random forest. PLoS One 7(9):e45854.

28. Yi L, et al. (2013) Engineering of TEV protease variants by yeast ER sequestration screening (YESS) of combinatorial libraries. Proc Natl Acad Sci U S A 110(18):7229-

34.

29. Shiryaev SA, et al. (2012) New details of HCV NS3/4A proteinase functionality revealed by a high-throughput cleavage assay. PLoS One 7(4):e35759.

30. Rögnvaldsson T, et al. (2009) How to find simple and accurate rules for viral protease cleavage specificities. BMC Bioinformatics 10:149.

31. Kostallas G, Löfdahl P-Å, Samuelson P (2011) Substrate profiling of tobacco etch virus protease using a novel fluorescence-assisted whole-cell assay. PLoS One 6(1):e16136.

32. Boulware KT, Jabaiah A, Daugherty PS (2010) Evolutionary optimization of peptide substrates for proteases that exhibit rapid hydrolysis kinetics. Biotechnol Bioeng 106(3):339-46. 
33. Ratnikov BI, et al. (2014) Basis for substrate recognition and distinction by matrix metalloproteinases. Proc Natl Acad Sci U S A 111(40):E4148-55.

34. Tyka MD, et al. (2011) Alternate states of proteins revealed by detailed energy landscape mapping. J Mol Biol 405(2):607-18.

35. London N, Lamphear CL, Hougland JL, Fierke CA, Schueler-Furman O (2011) Identification of a novel class of farnesylation targets by structure-based modeling of binding specificity. PLoS Comput Biol 7(10):e1002170.

36. Baugh EH, et al. (2016) Robust classification of protein variation using structural modelling and large-scale data integration. Nucleic Acids Res 44(6):2501-13.

37. Appadurai R, Senapati S (2016) Dynamical Network of HIV-1 Protease Mutants Reveals the Mechanism of Drug Resistance and Unhindered Activity. Biochemistry 55(10):1529-40.

38. Yi L, et al. (2015) Yeast Endoplasmic Reticulum Sequestration Screening for the Engineering of Proteases from Libraries Expressed in Yeast. Methods Mol Biol 1319:81-93.

39. Grakoui A, McCourt DW, Wychowski C, Feinstone SM, Rice CM (1993) Characterization of the hepatitis $\mathrm{C}$ virus-encoded serine proteinase: determination of proteinase-dependent polyprotein cleavage sites. J Virol 67(5):2832-43.

40. Grakoui A, Wychowski C, Lin C, Feinstone SM, Rice CM (1993) Expression and identification of hepatitis C virus polyprotein cleavage products. J Virol 67(3):138595.

41. Puente XS, Sánchez LM, Overall CM, López-Otín C (2003) Human and mouse proteases: a comparative genomic approach. Nat Rev Genet 4(7):544-58. 
42. Hou T, Zhang W, Case DA, Wang W (2008) Characterization of domain-peptide interaction interface: a case study on the amphiphysin-1 SH3 domain. J Mol Biol 376(4):1201-14.

43. Teyra J, Sidhu SS, Kim PM (2012) Elucidation of the binding preferences of peptide recognition modules: SH3 and PDZ domains. FEBS Lett 586(17):2631-7.

44. Li N, Hou T, Ding B, Wang W (2011) Characterization of PDZ domain-peptide interaction interface based on energetic patterns. Proteins 79(11):3208-20.

45. Smith CA, Kortemme T (2010) Structure-based prediction of the peptide sequence space recognized by natural and synthetic PDZ domains. J Mol Biol 402(2):460-74.

46. Crivelli JJ, Lemmon G, Kaufmann KW, Meiler J (2013) Simultaneous prediction of binding free energy and specificity for PDZ domain-peptide interactions. J Comput Aided Mol Des 27(12):1051-65.

47. Yanover C, Bradley P (2011) Large-scale characterization of peptide-MHC binding landscapes with structural simulations. Proc Natl Acad Sci U S A 108(17):6981-6.

48. Lanouette S, et al. (2015) Discovery of substrates for a SET domain lysine methyltransferase predicted by multistate computational protein design. Structure 23(1):206-15.

49. Chaudhury S, Gray JJ (2009) Identification of structural mechanisms of HIV-1 protease specificity using computational peptide docking: implications for drug resistance. Structure 17(12):1636-48.

50. Jensen JH, Willemoës M, Winther JR, De Vico L (2014) In silico prediction of mutant HIV-1 proteases cleaving a target sequence. PLoS One 9(5):e95833.

51. Smith CA, Kortemme T (2011) Predicting the tolerated sequences for proteins and 
protein interfaces using RosettaBackrub flexible backbone design. PLoS One 6(7):e20451.

52. Liu F, Luehr N, Kulik HJ, Martínez TJ (2015) Quantum Chemistry for Solvated Molecules on Graphical Processing Units Using Polarizable Continuum Models. J Chem Theory Comput 11(7):3131-44.

53. Romano KP, et al. (2012) The Molecular Basis of Drug Resistance against Hepatitis C Virus NS3/4A Protease Inhibitors. PLoS Pathog 8(7):e1002832.

54. Varadarajan N, Rodriguez S, Hwang B-Y, Georgiou G, Iverson BL (2008) Highly active and selective endopeptidases with programmed substrate specificities. Nat Chem Biol 4(5):290-4.

55. Khersonsky O, Tawfik DS (2010) Enzyme promiscuity: a mechanistic and evolutionary perspective. Annu Rev Biochem 79:471-505.

56. Rögnvaldsson T, et al. (2009) How to find simple and accurate rules for viral protease cleavage specificities. BMC Bioinformatics 10(1):149.

57. Ratnikov BI, et al. (2014) Basis for substrate recognition and distinction by matrix metalloproteinases. Proc Natl Acad Sci 111(40):E4148-E4155.

58. Igarashi Y, et al. (2007) CutDB: a proteolytic event database. Nucleic Acids Res 35(Database issue):D546-9.

59. Romano KP, Ali A, Royer WE, Schiffer CA (2010) Drug resistance against HCV NS3/4A inhibitors is defined by the balance of substrate recognition versus inhibitor binding. Proc Natl Acad Sci U S A 107(49):20986-91.

60. Prabu-Jeyabalan M, Nalivaika EA, King NM, Schiffer CA (2003) Viability of a drugresistant human immunodeficiency virus type 1 protease variant: structural insights 
for better antiviral therapy. J Virol 77(2):1306-15.

61. Waugh SM, Harris JL, Fletterick R, Craik CS (2000) The structure of the pro-apoptotic protease granzyme B reveals the molecular determinants of its specificity. Nat Struct Biol 7(9):762-5.

62. Phan J, et al. (2002) Structural basis for the substrate specificity of tobacco etch virus protease. J Biol Chem 277(52):50564-72.

63. Hashimoto H, et al. (2011) Structural basis for matrix metalloproteinase-2 (MMP-2)selective inhibitory action of $\beta$-amyloid precursor protein-derived inhibitor. J Biol Chem 286(38):33236-43.

64. Nivón LG, et al. (2013) A Pareto-Optimal Refinement Method for Protein Design Scaffolds. PLoS One 8(4):e59004.

65. Fleishman SJ, et al. (2011) RosettaScripts: A Scripting Language Interface to the Rosetta Macromolecular Modeling Suite. PLoS One 6(6):e20161.

66. Richter F, Leaver-Fay A, Khare SD, Bjelic S, Baker D (2011) De novo enzyme design using Rosetta3. PLoS One 6(5):e19230.

67. Miller BR, et al. (2012) MMPBSA.py : An Efficient Program for End-State Free Energy Calculations. J Chem Theory Comput 8(9):3314-3321.

68. Gront D, Kulp DW, Vernon RM, Strauss CEM, Baker D (2011) Generalized fragment picking in Rosetta: design, protocols and applications. PLoS One 6(8):e23294.

69. Bond SR, Naus CC (2012) RF-Cloning.org: an online tool for the design of restrictionfree cloning projects. Nucleic Acids Res 40(Web Server issue):W209-13. 


\section{Figure Legends:}

Figure 1. Overview of a general, energy-based discriminator An illustration of the mechanism of steps leading to the formation of a common tetrahedral intermediate (TI) for serine-, cysteine-, threonine (A), aspartic, glutamic (B), and metallo-proteases (C). Protease active site cleft is depicted as a dashed arc. (D) Generation of atomic resolution models of the near attack conformation using high resolution crystallographic structures and known cleaved and uncleaved sequence datasets. (E) The resulting complexes were allowed to relax into a minimum energy conformation using the described protocol (FastRelax) and scored using a linear combination of $(F)$ the sum of the interface residues' Rosetta energy (protease and peptide residue energies; two terms), (G) the sum of the interface residues' AMBER MMPBSA electrostatic scores, $(\mathrm{H})$ a score that describes the propensity of the peptide to adopt an extended conformation (reorganization penalty), and (I) the deviation of the active cleft residues from the idealized active conformations (a pseudo energy-term). The linear combination of weighted scores were recombined according to this equation: Total_score $=\mathrm{w} 1 *$ Rosetta_Interface_Energy $($ Protease energy $)+$ w2*Rosetta_Interface_Energy (Peptide energy) $+\mathrm{w} 3 *$ Catalytic constraint penalty $+\mathrm{w} 4$ ${ }^{*}$ Reorganization Penalty + w5* Electrostatic Binding Energy; where w1 =1, w2 =1, w3 = 3.5, $\mathrm{w} 4=0.01, \mathrm{w} 5=0.5$

Figure 2 Distribution of Discriminator Scores; Score distributions for cleaved sequences (depicted in black) and uncleaved (depicted in dotted bars) for (A) TEV protease (B) Granzyme B (C) HCV (D) HIV (E) MMP2. The $p$-values were calculated using a Wilcoxon rank test. A threshold based binary classification of sequences into cleaved and uncleaved 
sequences using these scores was performed and the auROC (F) values for the five proteases are indicated. (G) Enrichment of true cleaved sequences in the top-ranked pools. Enrichment ratio (black bars) = \#true cleaved/ \# of cleaved sequences in datatset. Background Enrichment (white bars), which represents fraction of cleaved sequences in the dataset, and Enrichment obtained from SitePrediction model (wavy bars) with $20 \%$ of the known cleaved sequences. In each case, the structure-based discriminator performs comparably or better than SitePrediction.

\section{Figure 3. Combining sequence and energy signatures leads to higher discriminatory}

power (A) The energetic features were used to train an SVM using a radial based function, which yielded higher auROC values for all proteases as compared to a linear combination of optimized weights. (B) auROCs obtained from support vector machines (SVMs) trained with sequence only(blue), energetic only(gray) and both sequence and energetic features(wavy) in a 5-fold cross-validation test. Black bars indicate auROC for the linear combination of weighted score terms. The combination of sequence and energy features consistently results in higher auROC values. (C) Accuracy as a function of training set size used for training for the (C) sequence, (D) energetic features, and (E) both sequence and energetic features. The accuracy values are not altered appreciably when a significantly smaller training dataset is used. In-set classification and generalization curves converge as a progressively higher fraction of the dataset is used for training. The classification curve is shown in red whereas the generalization curve is depicted in blue. 


\section{Fig. 4. Multi-body interaction networks at the interface underlie improved}

discrimination. Several sequences are misclassified by the Sequence-Based Discriminator whereas they are correctly classified when the Structure based Discriminator is used. (A) The sequence 'GPGTARSPP' is misclassified by the sequence based SVM as 'cleaved' for the HIV protease sequence set. Residue P3' of the peptide is packed in the vicinity of ARG 8; which is involved in a key interaction with ASP 29 necessary in maintaining the dimer interface. The P3' -ARG 8 repulsion leads to a destabilization of one of the key interactions involved in dimer interface stabilization. One half of dimer surface is shown as a cartoon representation and the other as a charged surface in order to highlight the dimer interface of the HIV protease. This electrostatic repulsion is captured by the energy-based approach but not the sequence based approach, leading to misclassification by the latter (B) 'SQAYPIVQ' is misclassified as an uncleaved sequence present in the HIV protease sequence set. The P1 tyrosine residue (yellow) along with the serine at P4 forms a favorable hydrogen bond network with ARG 8 (green) allowing for substrate cleavage. This favorable hydrogen-bonding network is likely not directly recognized by the sequence-based approach. (C) 'KPAIIPDR' belongs to the HCV Protease sequence set which is misclassified as cleaved by the sequence-based approach. The presence of proline at positions P5 and P1(yellow) bends the substrate chain in an orientation that is unfavorable for cleavage. The extended conformation of a peptide, which allows hydrogen bond formation, leading to binding of the peptide and eventually cleavage, is highlighted (purple). The Rosetta energies correctly detect this disruption of the hydrogen bond network caused by the presence of proline residues between peptide (yellow) and protease. 
Figure 5. Discovering novel sequence specificities HCV NS3 4A Protease (A) Sequence Logo plots of the identified four novel sequence motifs whose scores overlapped with the cleaved sequences in the benchmark dataset (B) Schematic of the vector (LY104) used for the YESS assay. The vector contains Aga2 cell surface signaling moiety followed by the substrate flanked between HA tag and FLAG tag which can be detected on the cell surface by fluorescently tagged antibodies. The protease and substrate are co-expressed in the ER of the yeast cell. If cleavage occurs the FLAG:HA ratio is 0 , if substrate is uncleaved ratio is 1. (C) Results of the YESS assay test of the predicted cleaved sequences. Three out of the four tested sequences (predicted cleaved; green bar) showed a FLAG:HA ratio $<0.5$. The positive control (wild type shown in blue) showed an expected low FLAG/HA ratio whereas the negative control (known and predicted uncleaved sequences, red bars) showed high FLAG:HA ratios $>0.85$. The protease knockout mutant S139A (dotted red bars) showed FLAG:HA ratio $>0.85$ for all sequences, confirming that the sequences were cleaved because of the co-expressed HCV NS3 protease from the assay vector and not an endogenous yeast ER enzyme. (D) Cell cytometry histograms of LEEFFCSG, predicted cleaved sequence showing a $62.1 \%$ cell population signal for HA tag, $11.4 \%$ cell population signal for FLAG, thus showing a FLAG:HA ratio of 0.18 (E) Cell cytometry histograms for the negative control sequence DKNQVEGE, showing a 38.3\% cell population signal for HA tag, and $34.0 \%$ for FLAG tag, thus exhibiting a FLAG:HA ratio of 0.88 .

\author{
Figure 6: Summary of cleaved and uncleaved dataset distributions, model generation \\ and active site geometry of the starting crystal structure and the primary biophysical \\ mode of recognition of proteases considered in the study (A) HCV Protease (PDB ID:
}


$3 \mathrm{M} 5 \mathrm{~N}$ ), a serine protease shows recognition via interfacial hydrogen bonding. (B) Granzyme B (PDB ID:1FI8) a serine protease shows an electrostatic mode of substrate recognition (C) TEV Protease, (PDB ID:1LVB), a cysteine protease displaying extensive hydrogen bonding at the protease-substrate interface (E) HIV Protease I (PDB ID: 1MT9), a symmetric homodimeric aspartyl protease, where recognition appears to be primarily sterics-based. (F) MMP2 (PDB ID: 3AYU), a zinc catalytic center. 


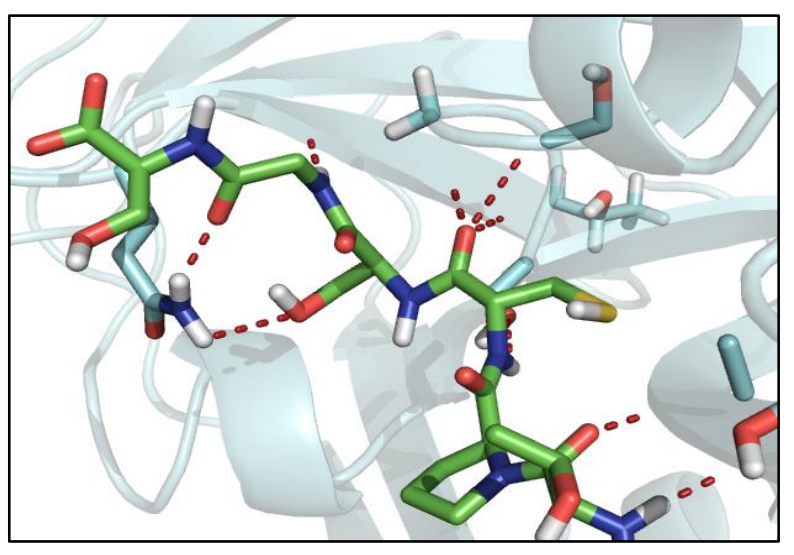

Interface Residue Energy

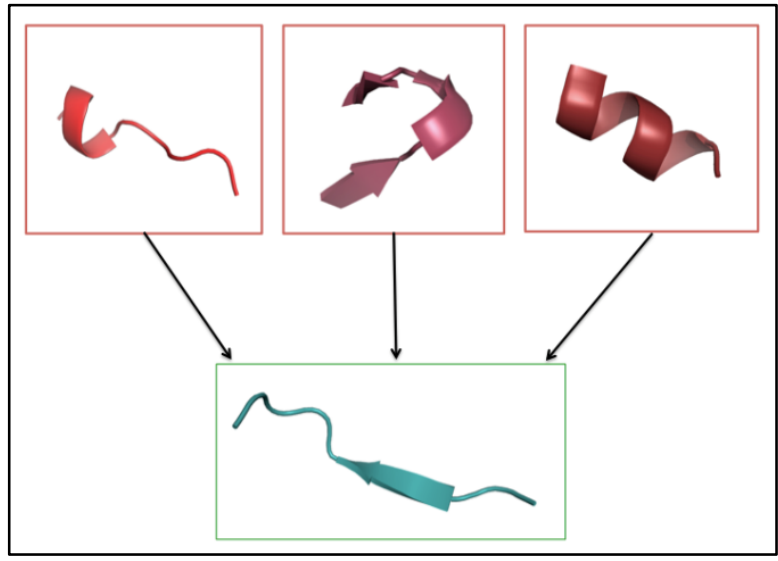

Reorganization Penalty

(Propensity of unbound peptide to form bound structure)
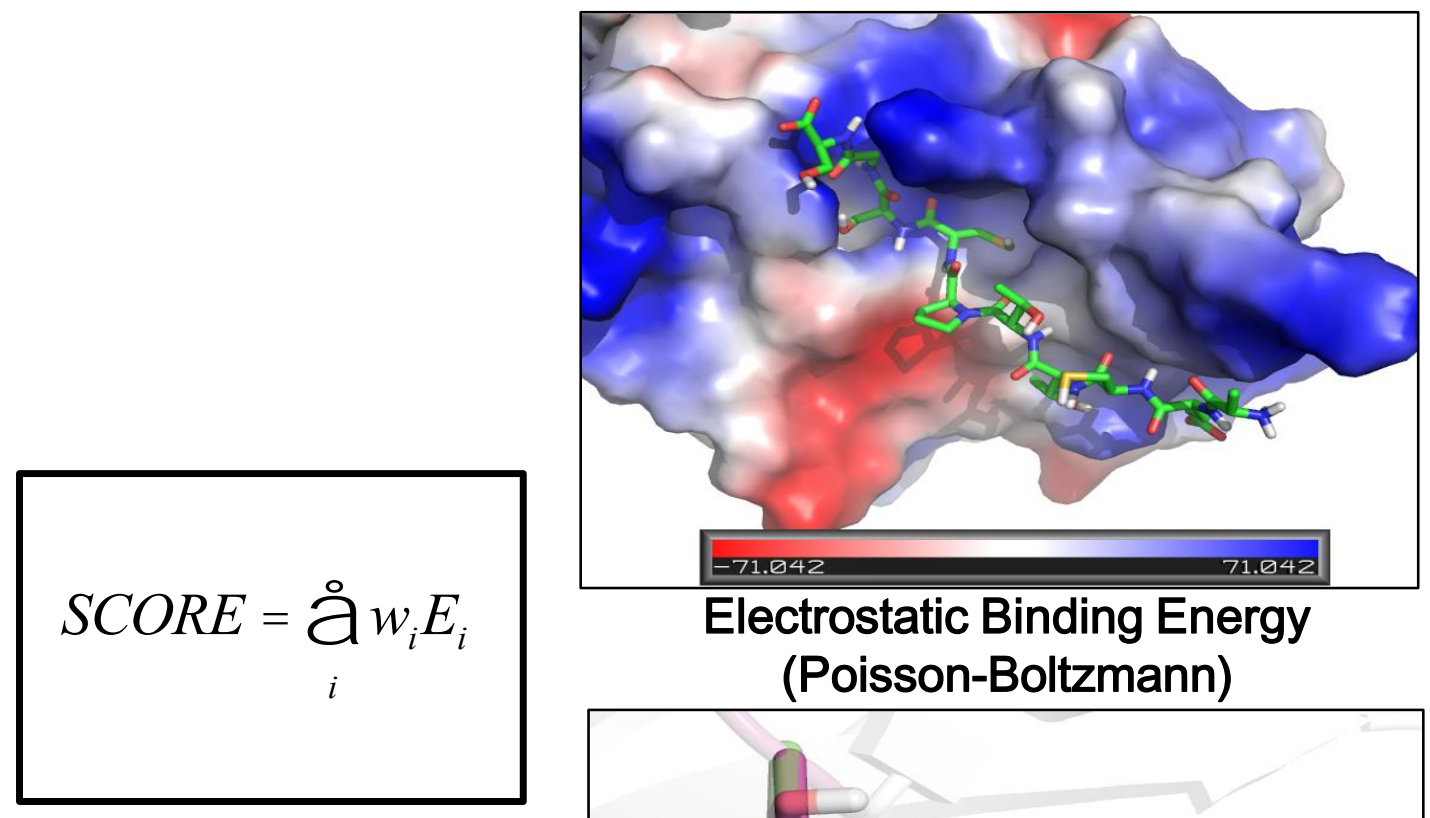

\section{Electrostatic Binding Energy}

(Poisson-Boltzmann)

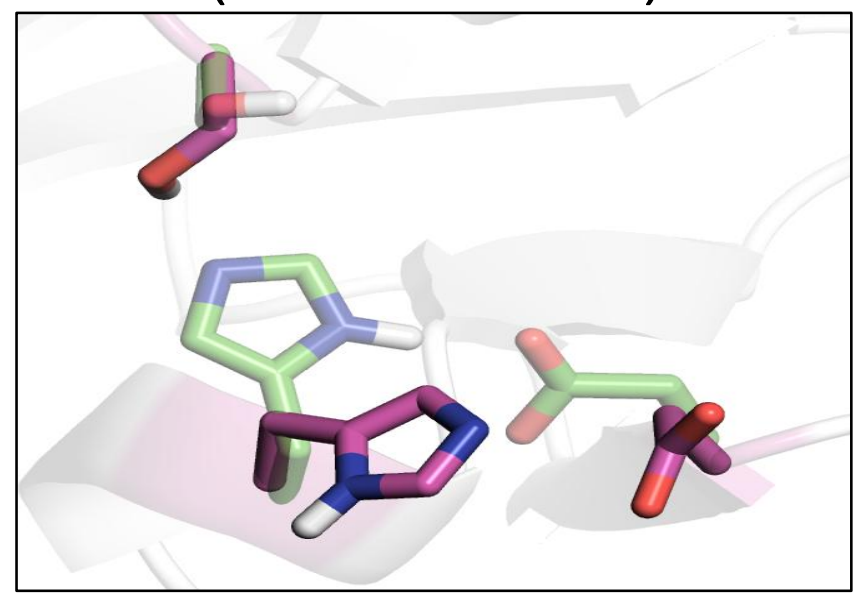

Geometric Constraints 


\section{Main text: Tables}

\section{Table 1: Details of starting Model Generation for five proteases}

\begin{tabular}{|c|c|c|c|}
\hline Protease & PDB ID & Resolution & Model Generation \\
\hline $\begin{array}{l}\text { HCV NS3 } \\
\text { Protease }\end{array}$ & $\begin{array}{l}3 \text { M5L, } \\
3 \text { M5N }\end{array}$ & $1.9 \AA$ & $\begin{array}{l}\text { The P' residues of the bound peptide were } \\
\text { built by overlaying PDB ID: 3M5N and PDB } \\
\text { ID:3M5L (inhibitor bound crystal structure) } \\
\text { thus allowing us to build a complete } \\
\text { substrate bound complex }\end{array}$ \\
\hline TEV Protease & $\begin{array}{l}1 \mathrm{LVB}, \\
1 \mathrm{LVM}\end{array}$ & $2.2 \AA$ & $\begin{array}{l}\text { Starting model generated from PDB by } \\
\text { reverting C151A to WT }\end{array}$ \\
\hline MMP2 & $\begin{array}{l}\text { 3AYU, } \\
1 \mathrm{BQQ}\end{array}$ & $2.0 \AA$ & $\begin{array}{l}\text { Starting model was generated by } \\
\text { superimposing PDB ID: 1BQQ with PDB } \\
\text { ID:3AYU(MMP2). The N terminal (P side) } \\
\text { residues of the substrate were extended } \\
\text { outward to build the complete substrate } \\
\text { and were then relaxed to find an optimal } \\
\text { substrate conformation }\end{array}$ \\
\hline Granzyme B & 1FI8 & $2.2 \AA$ & $\begin{array}{l}\text { The interface of the ecotin chain in the } \\
\text { crystal structure, spanning eight residue } \\
\text { substrate chain was used as the starting } \\
\text { point for further calculations }\end{array}$ \\
\hline HIV Protease 1 & 1MT9 & $2.0 \AA$ & $\begin{array}{l}\text { Starting model generated by inverting } \\
\text { D25N and V82N from crystal structure to } \\
\text { native residue identities }\end{array}$ \\
\hline
\end{tabular}



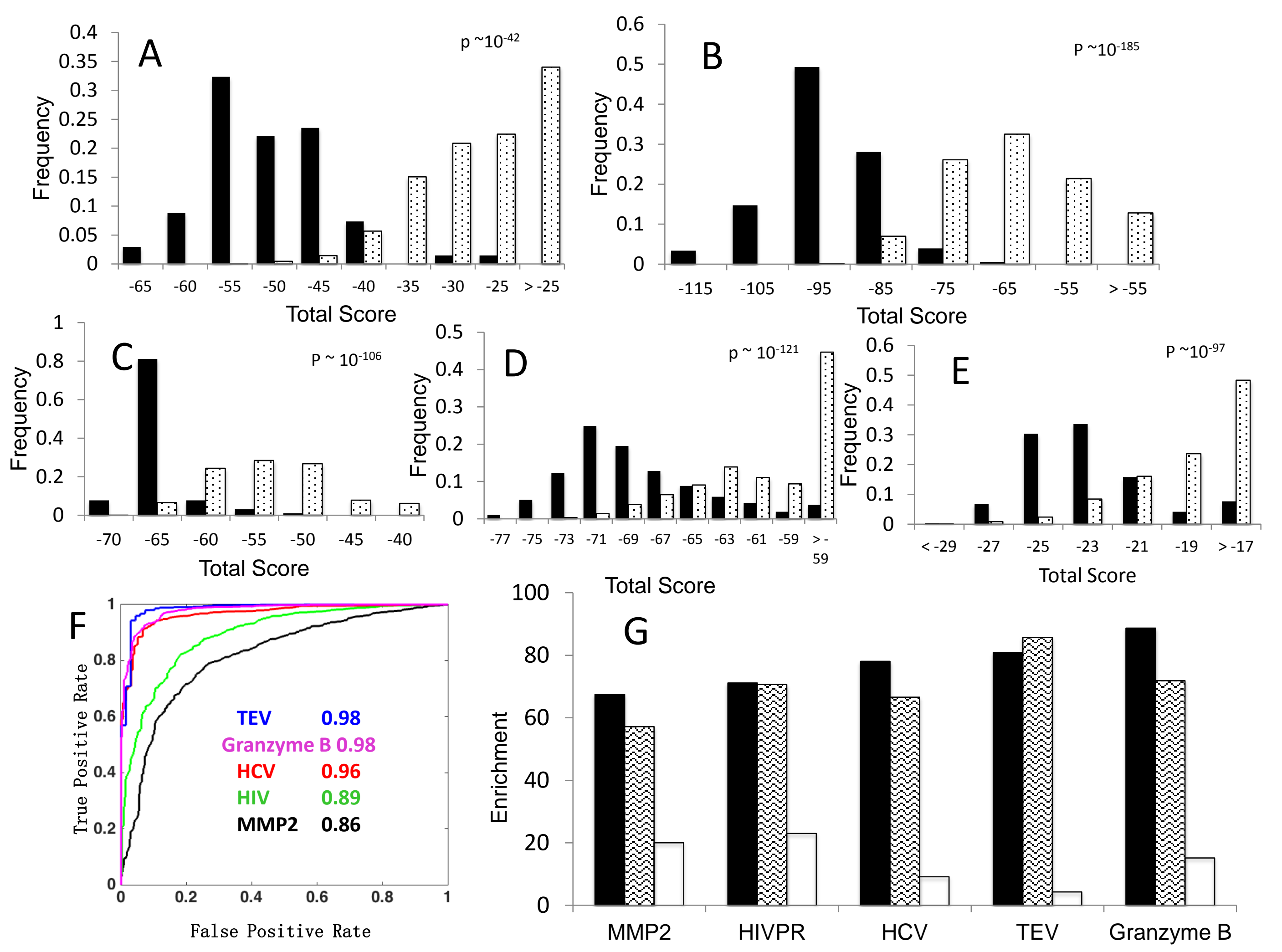

G 

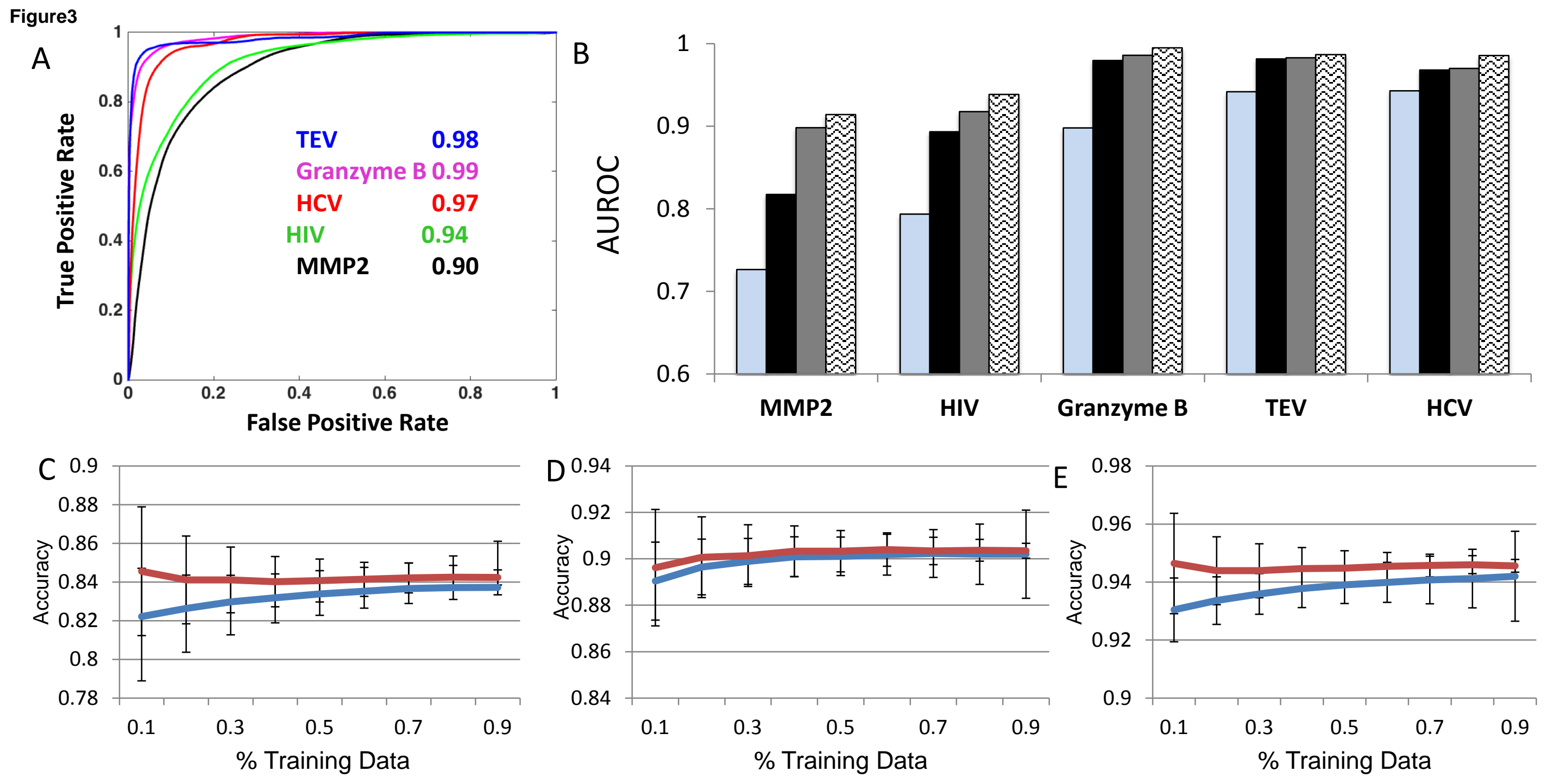


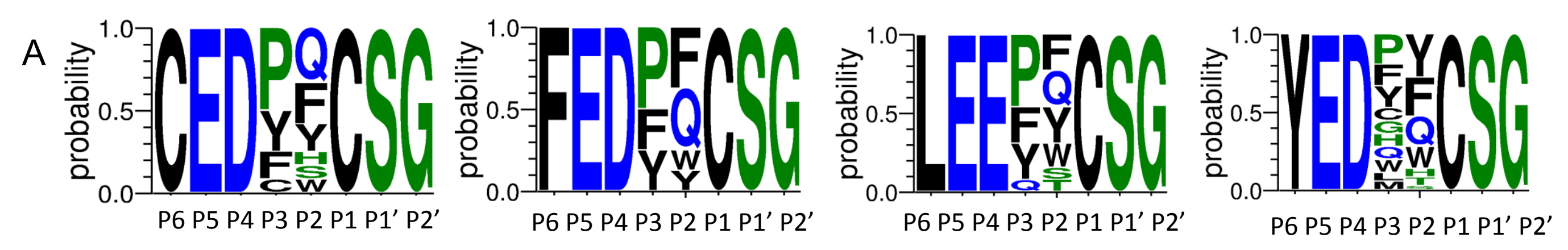

B
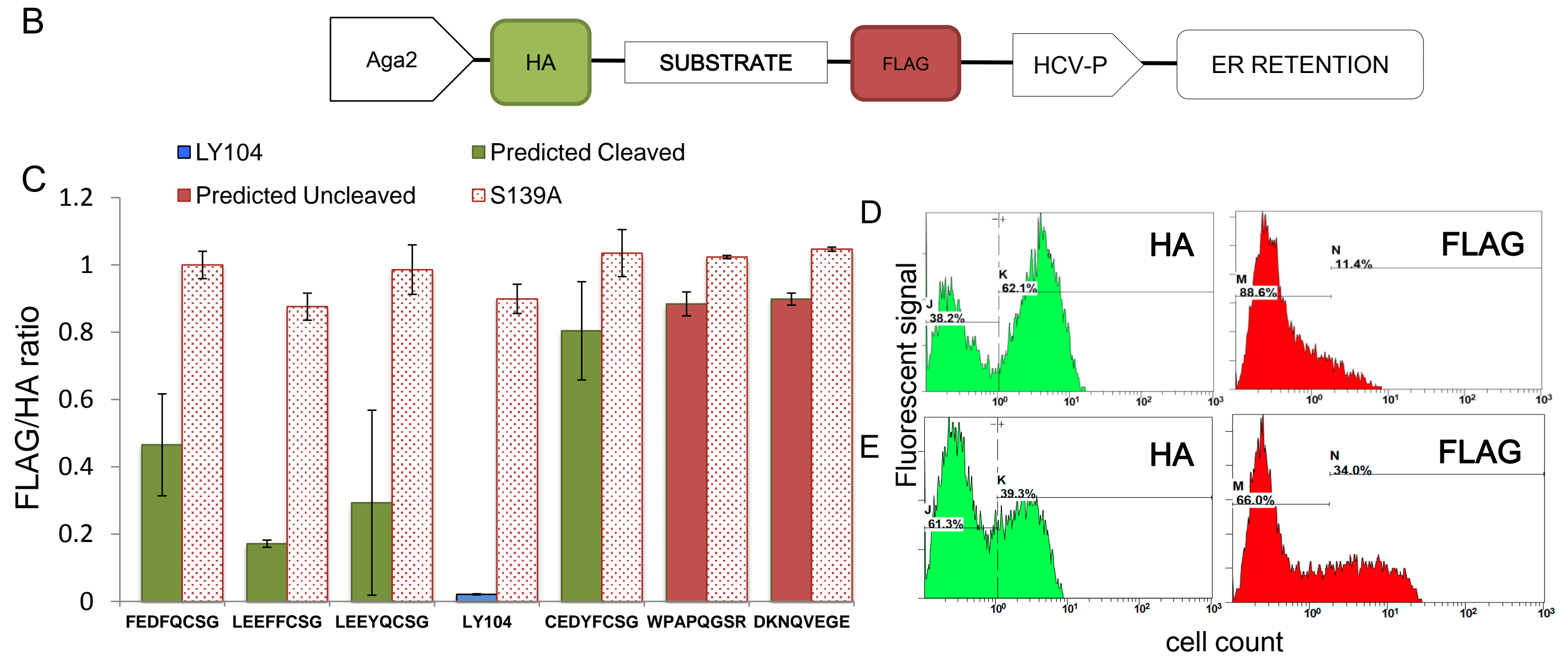


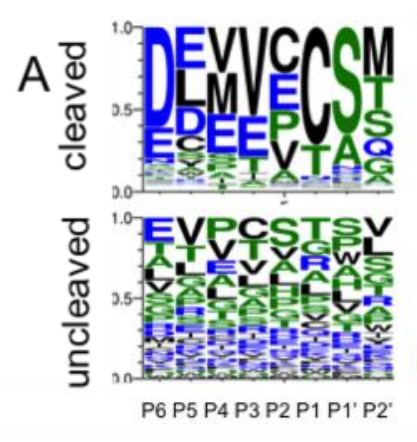

$B$

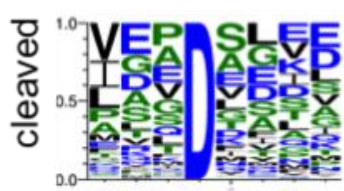

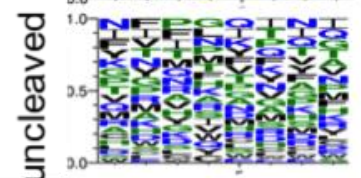

PAP P P P P'P P P P P P4

C

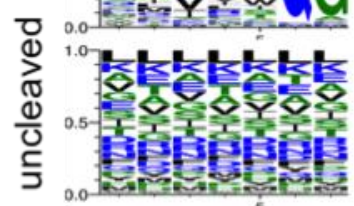

P6 P5 P4 P3 P2 P1 P1

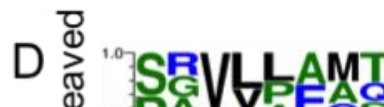

o. pis

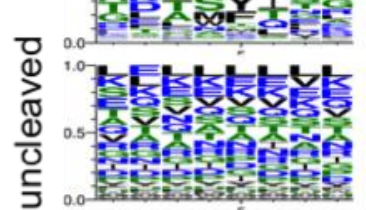

P4 P3 P2 P1 P1' P2' P3' P4

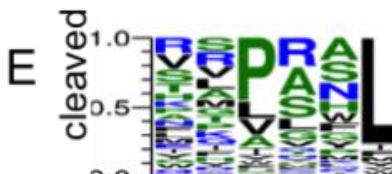

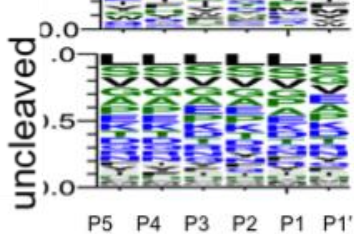

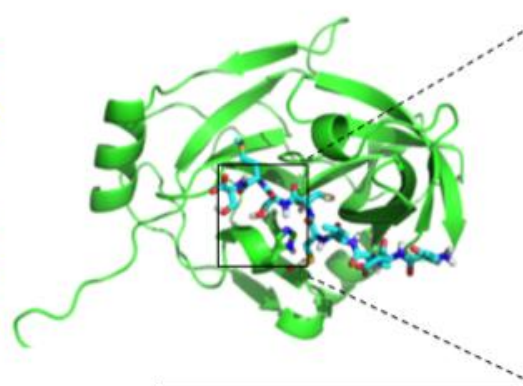
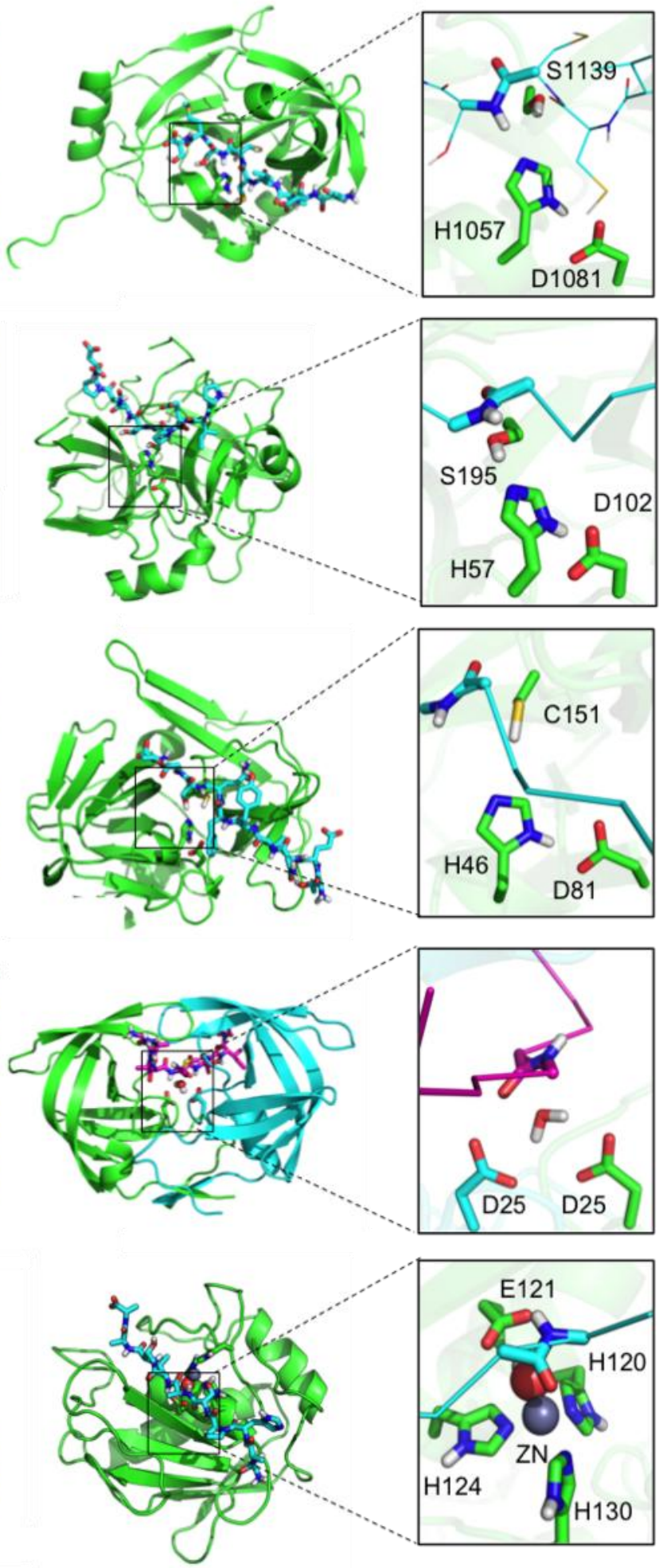
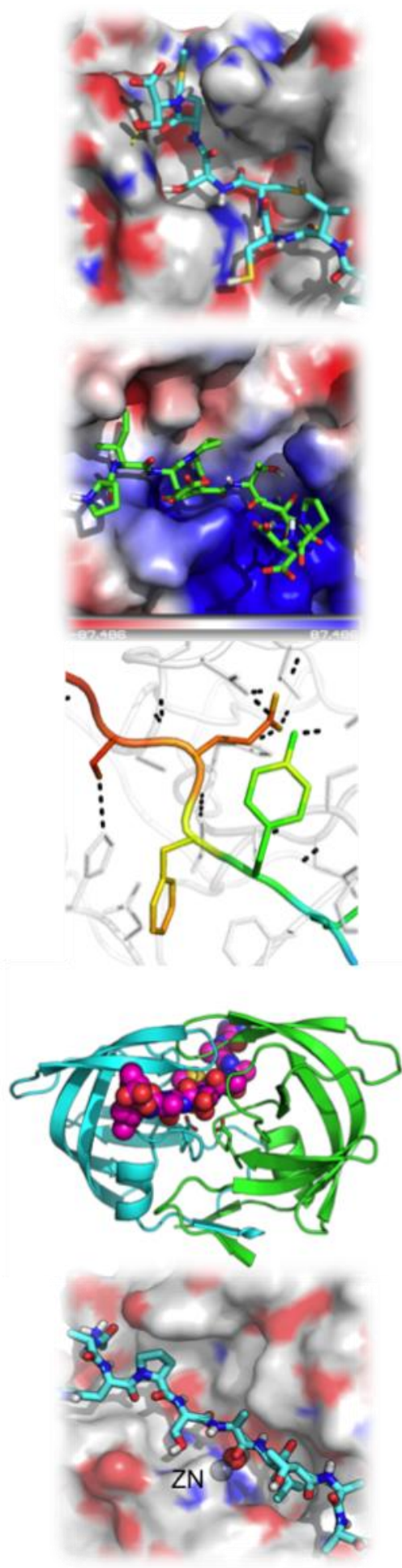